\title{
Polarimetric observations of comet 67P/Churyumov-Gerasimenko during its 2008-2009 apparition ${ }^{\star}$
}

\author{
E. Hadamcik ${ }^{1}$, A. K. Sen ${ }^{2}$, A. C. Levasseur-Regourd ${ }^{1}$, R. Gupta ${ }^{3}$, and J. Lasue ${ }^{4}$ \\ 1 UPMC Univ. Paris 06 / LATMOS-IPSL, 11 bld d'Alembert, 78280 Guyancourt, France \\ e-mail: [edith.hadamcik; aclr]@latmos.ipsl.fr \\ 2 Assam Univ., Silchar 788001, India \\ e-mail: asokesen@yahoo.com \\ 3 IUCAA, Post Bag 4, Ganeshkhind, Pune-411007, India \\ e-mail: rag@iucaa.ernet.in \\ ${ }^{4}$ LPI, 3600 Bay Area Blvd, Houston, TX 77058-1113, USA \\ e-mail: lasue@lpi.usra.edu \\ Received 31 January 2010 / Accepted 12 April 2010
}

ABSTRACT

\begin{abstract}
Context. Remote observations of the light scattered by comet 67P/Churyumov-Gerasimenko dust coma are of major importance for determining the physical properties of the particles and prepare the rendezvous with the ESA/Rosetta spacecraft in 2014.

Aims. Light scattering and especially linear polarization observations allow comparison between different coma regions and different comets, including comets that have been studied by space probes. Our aim is to retrieve physical properties of the dust particles and to characterize their evolution around perihelion passage.

Methods. Recent imaging polarimetric observations were conducted at the Haute-Provence observatory (France) on 2009 March $17-19$ at $35^{\circ}$ phase angle and at IUCAA Girawali observatory (India) on 2008 December $25-27$ at $36^{\circ}$ phase angle and on 2009 April 30-May 1 at $29^{\circ}$ phase angle. With the imaging technique, the intensity and linear polarization variations are studied through the various coma regions. These observations are compared to other cometary data (e.g. Jupiter family comets) and to numerical and experimental simulations.

Results. The decrease in intensity as a function of the distance to nucleus in log-log scale is on average close to -1 , although important variations with values down to -1.5 are noticed, in agreement with previous observations in 1982-83 and 1995-96. The intensity along the tailward direction decreases with a slope between -1.2 two months before perihelion (2009 February 28 ) to -1.0 two months after perihelion, and the decrease is more pronounced in the sunward direction. Before perihelion, aperture polarization values are comparable to polarization values measured on other comets at similar phase angles. The sharp decrease in intensity and the feature in the tailward direction, without any difference in polarization in the coma before perihelion, could suggest the presence of large dark particles. The post-perihelion increase in intensity and in polarization suggests that an outburst has occurred. The freshly ejected dust polarizes more the scattered light and is more sensitive to the solar radiation pressure, suggesting small micron- or submicron-sized grains.

Conclusions. Polarization and intensity variations in the coma of 67P/Churyumov-Gerasimenko are reminiscent of those noticed for some comets such as comet 81P/Wild 2 and comet 9P/Tempel 1. The presence of rather large particles can thus be suggested before and just after perihelion and the ejection of post-perihelion smaller grains, eventually in fluffy aggregates. A strong seasonal effect related to the obliquity of the comet suggests that the different grains originate in different hemispheres of the nucleus.
\end{abstract}

Key words. comets: individual: 67P/Churyumov-Gerasimenko - polarization - scattering - techniques: polarimetric

\section{Introduction}

Comet 67P/Churyumov-Gerasimenko (hereafter 67P/C-G) is the target of the European Space Agency's Rosetta mission. The spacecraft was launched in 2004 and is scheduled to rendezvous with 67P/C-G in 2014 (Glassmeier et al. 2007) when the comet will be relatively inactive, at about a heliocentric distance $d_{s}$ between 3.8 AU and 3.5 AU. The Rosetta spacecraft will progressively approach the nucleus to release the Philae lander at about $d_{\mathrm{s}}=3.1 \mathrm{AU}$ and significantly before the perihelion, which will take place by 2015 August. The spacecraft will observe the comet along its orbit through the perihelion and monitor the increase of cometary activity. The maximum activity is expected

* Based in part on observations made at Observatoire of HauteProvence (CNRS), France and in part at IUCAA Girawali observatory, India. about one month after perihelion as for previous apparitions (Ferrin 2005). The mission should end in 2015 December when the comet will still be active.

Comet $67 \mathrm{P} / \mathrm{C}-\mathrm{G}$ was discovered in 1969 . Its orbital history indicates two encounters with Jupiter leading to a perihelion distance of $1.245 \mathrm{AU}$ and an orbital period of 6.44 years (Rocher 2009). Since its discovery, it was observed at each approach and found to be among the relatively active Jupiter family comets. It is classified as a dusty comet and is slightly $\mathrm{C}_{2}$-to$\mathrm{CN}$ depleted in the A'Hearn et al. (1995) database. Schleicher (2006) recalculated the $\mathrm{C}_{2}$-to-CN ratios and qualified 67P/C-G as mildly depleted. Using the Planetary Camera of the Hubble Space Telescope, Lamy et al. (2007) measured a rotational nucleus period of $(12.3 \pm 0.27) \mathrm{h}$. From Spitzer Space Telescope observations, Lamy et al. (2008) derived an effective radius (for a sphere of equivalent volume) of about $2 \mathrm{~km}$ and an albedo of 
0.04 for the irregular nucleus. Davidsson and Gutiérrez (2005) derived a nucleus bulk density in the range $100-370 \mathrm{~kg} \mathrm{~m}^{-3}$ from non-gravitational forces studies. Tail, trails, and neckline were observed at different apparitions (Fulle 2004; Moreno et al. 2004; Agarwal 2007; Kelley et al. 2008; Ishiguro 2008). All these authors suggest there are very large $\mathrm{mm}$ to $\mathrm{cm}$-sized particles pre-perihelion.

Linear polarization studies, which are independent of the number of particles in the field of view (for optically thin media), give complementary indications for the optical and physical properties inside the coma (see e.g. Levasseur-Regourd 1999). Previous observations through similar polarimetric imaging methods (Sen et al. 1990; Renard et al. 1992; Hadamcik et al. 2003a) have allowed the different regions in the coma to be confirmed. The different physical properties of the particles are indicated by the value of the polarization, as pointed out by in-situ observations in the coma of comet 1P/Halley (LevasseurRegourd et al. 1986, 1999a). By using successive images of comet C/1995 O1 (Hale-Bopp), the evolution of structures in the coma has been followed during hours, days, and months (Hadamcik \& Levasseur-Regourd 2003b). In the jets and arcs, the polarization was higher than in the surrounding coma, indicating smaller grains and/or fluffier particles. The coma of comet C/1999 S4 (LINEAR) was observed during the complete disruption of its nucleus. Variations in the polarization associated with intensity variations allowed us to characterize large fragmenting compact particles (Hadamcik \& Levasseur-Regourd 2003c). The polarization measured in the coma of comet 9P/Tempel 1, has shown variations correlated to the period of rotation of the nucleus before impact and different properties of the dust ejected by the impact (Hadamcik et al. 2007a). To interpret the polarization and intensity variations, numerical and experimental simulations are currently being used (Lasue et al. 2009; Hadamcik et al. 2006, 2007b, 2009).

Preliminary results about 2008-2009 observations of 67P/C-G can be found in Levasseur-Regourd et al. (2010). In Sect. 2, we describe the two instruments and their data reduction procedure. In Sect. 3, we present the results for the three observing periods and emphasize the coma asymmetries by a detailed study of the intensity and polarization variations. In Sect. 4, the results are compared to those of previous apparitions and to the results for other comets, especially for other Jupiter family comets (hereafter JFCs) and more active comets, leading to some clues about the evolution of the physical properties in the coma.

\section{Observations and data reduction}

Two different telescopes were used through a France-India collaboration. The observations were conducted at Observatoire de Haute Provence (OHP) near Marseille in France and at the InterUniversity Center for Astronomy and Astrophysics (IUCAA Girawali Observatory, "IGO”) near Pune in India.

\subsection{Instruments}

2.1.1. OHP Haute-Provence Observatory, France $\left(43^{\circ} 55^{\prime} 54^{\prime \prime}\right.$ $\mathrm{N}$ latitude, $5^{\circ} 42^{\prime} 44^{\prime \prime}$ E longitude, altitude $650 \mathrm{~m}$ )

The OHP $80 \mathrm{~cm}$ telescope is opened at $f / 15$ in Cassegrain mode with a field of 7 arcmin on the side. The CCD camera has $2048 \times$ 2048 pixels, back-illuminated, of $13.5 \mu \mathrm{m}$ each. The resolution by pixel is thus 0.21 arcsec. A current binning of $4 \times 4$ pixels was applied. The CCD is cooled down to $-50{ }^{\circ} \mathrm{C}$ by a 5 -stage Peltier system.

A red filter is used to avoid contaminations by the gaseous species ( $\operatorname{red} R$, centered on $\lambda=650 \mathrm{~nm}, \Delta \lambda=90 \mathrm{~nm})$. A residual contamination by the gaseous species (mainly $\mathrm{NH}_{2}$ and [OI]) is possible but seems negligible for this dusty comet (justification in Sect. 3.2.2). The telescope tracking-mode is stellar. Very short (20 s) exposure times are needed to avoid a noticeable movement of the photocenter during exposures and changes in the sky background from one image to the next one.

Four polaroid filters are mounted on a rotating wheel, with their fast axis oriented at $45^{\circ}$ from one another, the first one corresponding to the so-called direction Zero " 0 ". For each orientation, a polarized intensity image is recorded (so-called $Z_{0}, Z_{45}$, $Z_{90}, Z_{135}$ ), and $Z_{i}$ represents the polarized image, the integrated intensity measured through an aperture or the polarized intensity recorded by one pixel. The intensity $Z$, the linear polarization $P$, and the angle $\theta$ between the direction of polarization and the direction of the fast axis of filter 0 in the plane of observation are calculated through

$$
\begin{aligned}
& Z=Z_{0}+Z_{90}=Z_{45}+Z_{135} \\
& P=200 \frac{\sqrt{\left(Z_{0}-Z_{90}\right)^{2}+\left(Z_{45}-Z_{135}\right)^{2}}}{Z_{0}+Z_{90}+Z_{45}+Z_{135}} \\
& \theta=\frac{\left(Z_{45}-Z_{135}\right)}{\left(Z_{0}-Z_{90}\right)}
\end{aligned}
$$

\subsubsection{IGO, India $\left(19^{\circ} 5^{\prime} \mathrm{N}\right.$ latitude, $73^{\circ} 40^{\prime}$ longitude, altitude $1000 \mathrm{~m}$ )}

The IGO $200 \mathrm{~cm}$ telescope is opened at $f / 10$ in Cassegrain mode. With the IFOSC instrument (IUCAA Faint Object Spectrograph and Camera), the spatial sampling scale at the detector is $44 \mu \mathrm{m}$ per arcsec, giving a field of 10.5 arcmin on the side. The field of view for imaging polarimetry is reduced to about 2 arcmin radius. The CCD is back-illuminated with $13.5 \mu \mathrm{m}$-sized pixels, corresponding to a resolution of 0.307 arcsec by pixel. The CCD is cooled by liquid nitrogen.

To avoid contaminations by gaseous species, two narrowband ESA filters are used (blue CB $\lambda=443 \mathrm{~nm}, \Delta \lambda=4 \mathrm{~nm}$; red CR $\lambda=684 \mathrm{~nm}, \Delta \lambda=9 \mathrm{~nm}$ ) and a broadband Bessel red filter $\left(R_{\mathrm{b}} \lambda=630 \mathrm{~nm}, \Delta \lambda=120 \mathrm{~nm}\right)$. Through this last filter, some contamination may exist, but as stated for the $R$ filter used at OHP, it seems to be negligible (see justification in Sect. 3.2.1). The telescope tracking-mode is first stellar because of technical problems (that need short exposures) and finally cometary by differential tracking. The exposure times can be found in Table 1.

A rotating half-wave plate (HWP) with its fast axis normal to the optical axis of the system is associated to a Wollaston prism. At each position of the plate, two orthogonally polarized beams are produced. The separation between the ordinary and extraordinary components is 0.8 arcmin. Two successive positions of the half-wave plate are needed to retrieve the degree of polarization and the position angle of the polarization vector. More details on the principles of the instrument are described in Ramaprakash et al. (1998). When the half-wave plate rotates successively $22.5^{\circ}, 45^{\circ}$, and $67.5^{\circ}$ from an initial position denoted 0 , the position angles of the polarized components respectively rotate $45^{\circ}, 90^{\circ}$, and $135^{\circ}$ from the initial position. As for the OHP images, the images on each CCD plate are denoted $Z_{0}$, $Z_{90}$ (position 0 of HWP), $Z_{45}, Z_{135}$ (position 22.5 of HWP), $Z_{90}$, $Z_{0}$ (position 45 of HWP), $Z_{135}$, and $Z_{45}$ (position 67.5 of HWP). 
Table 1. Log of the observations ${ }^{a}$.

\begin{tabular}{|c|c|c|c|c|c|c|c|c|c|c|}
\hline $\begin{array}{c}\text { Date } \\
2008-2009\end{array}$ & $\begin{array}{c}\text { Days to } \\
\text { perihelion }\end{array}$ & $\begin{array}{c}\Delta \\
(\mathrm{AU})\end{array}$ & $\begin{array}{c}R_{\mathrm{S}} \\
(\mathrm{AU})\end{array}$ & $m_{\mathrm{v}}$ & $\begin{array}{c}\text { Alpha } \\
\left({ }^{\circ}\right)\end{array}$ & $\begin{array}{l}\text { Sun-C } \\
\text { PA }\left(^{\circ}\right)\end{array}$ & Telescope & $\begin{array}{c}D \\
\left(\mathrm{~km} \mathrm{px}^{-1}\right)\end{array}$ & Filters & $\begin{array}{c}\text { Exposure } \\
\text { time }\end{array}$ \\
\hline \multicolumn{11}{|l|}{ December } \\
\hline 25.60 & -65 & 1.67 & 1.45 & 14.2 & 35.75 & 70.5 & & 370 & $\mathrm{CR}$ & $180 \mathrm{~s}$ \\
\hline 26.62 & -64 & 1.67 & 1.46 & 14.2 & 35.75 & 70.5 & IGO & 373 & CR & $120 \mathrm{~s} \times 2$ \\
\hline 27.59 & -63 & 1.67 & 1.46 & 14.2 & 35.8 & 70.4 & (India) & 373 & $\mathrm{CB}$ & $240 \mathrm{~s} \times 2$ \\
\hline 27.61 & -63 & 1.67 & 1.46 & 14.2 & 35.8 & 70.3 & $2 \mathrm{~m}$ & 373 & CR & $180 \mathrm{~s} \times 2$ \\
\hline 27.63 & -63 & 1.67 & 1.46 & 14.2 & 35.8 & 70.3 & & 373 & $R_{\mathrm{b}}$ & $60 \mathrm{~s} \times 2$ \\
\hline \multicolumn{11}{|l|}{ March } \\
\hline 17.81 & +17 & 1.72 & 1.26 & 13.5 & 34.7 & 69.9 & OHP & 1051 & $R$ & $20 \mathrm{~s} \times 9$ \\
\hline 18.78 & +18 & 1.72 & 1.265 & 13.5 & 34.7 & 70.0 & (France) & 1057 & $\mathrm{R}$ & $20 \mathrm{~s} \times 18$ \\
\hline 19.80 & +19 & 1.73 & 1.27 & 13.5 & 34.6 & 70.2 & $0.8 \mathrm{~m}$ & 1057 & $R$ & $20 \mathrm{~s} \times 21$ \\
\hline \multicolumn{11}{|l|}{ April-May } \\
\hline 30.60 & +61 & 1.99 & 1.44 & 14.5 & 28.9 & 82.3 & IGO & 445 & $R_{\mathrm{b}}$ & $360 \mathrm{~s}$ \\
\hline 01.70 & +62 & 2.00 & 1.45 & 14.5 & 28.7 & 82.7 & $2 \mathrm{~m}$ & 445 & $R_{\mathrm{b}}$ & $600 \mathrm{~s}$ \\
\hline
\end{tabular}

Notes. ${ }^{(a)} \Delta$ = Earth-comet distance; $R_{\mathrm{S}}=$ Sun-comet distance; $m_{\mathrm{v}}=$ expected magnitudes given by the JPL Horizons system ephemeris; $\alpha=$ phase angle; Sun-C PA = extended Sun-comet radius vector position angle; $D=$ projected diameter for 1 pixel; filters: $\mathrm{CR}=$ narrow cometary red filter, $\mathrm{CB}=$ narrow cometary blue filter, $R_{\mathrm{b}}=$ broad red filter centered on $620 \mathrm{~nm}, R=$ broad red filter centered on $650 \mathrm{~nm}$ (see Sect. 2.1). Exposure time for each polarized component image $\times$ number of images used for the results.

The polarization degree $P$ and the position angle $\theta$ of the polarization vector only depend on ratios of polarized intensities measured on the same plate without any necessary normalization between the plates (sky transparencies, exposure times). If $\beta$ is the position of HWP and $R(\beta)$ a ratio defined by

$R(\beta)=\frac{I_{\mathrm{e}}(\beta) / I_{\mathrm{o}}(\beta)-1}{I_{\mathrm{e}}(\beta) / I_{\mathrm{o}}(\beta)+1}$

with $I_{\mathrm{e}}(\theta)$ and $I_{\mathrm{o}}(\theta)$ respectively the extraordinary and ordinary image on the same plate

$P=\sqrt{R(\beta)^{2}+R(\beta+22.5)^{2}}$

$\theta=0.5 \arctan \left(\frac{R(\beta+22.5)}{R(\beta)}\right)$.

The normalization of the sky background and the stability of the total intensity $\left(Z=Z_{0}+Z_{90}=Z_{45}+Z_{135}\right)$ are mandatory for building a polarization map (exposure times, if different between images have to be normalized first). A minimum series of four plates is required to control the stability of the sky background (mainly when the object is low on the local horizon). The four polarized components have to be separated and centered to build the total intensity image and the polarization map.

\subsection{Data reduction}

Each individual image was corrected for bias and flat field. To control that errors were not included in polarization values owing to the averaging of the intensity on the field, calculations were made with and without flat-field correction. The difference was negligible in comparison with other errors. The sky background was estimated in a region outside of the coma and free of faint stars (usually between the two polarized images of the same plate for IGO observations). A gravity center algorithm method was used to find the position of the photocenter of the comet on each polarized image. Only the brighter pixels close to the photocenter (typically 16) were taken into account. To avoid artifacts, the images were centered with a precision of 0.1 pixel. For the April-May observations at IGO, the cometary differential tracking with relatively long exposure times due to the faintness of the comet made it difficult to have such precision on the centering.

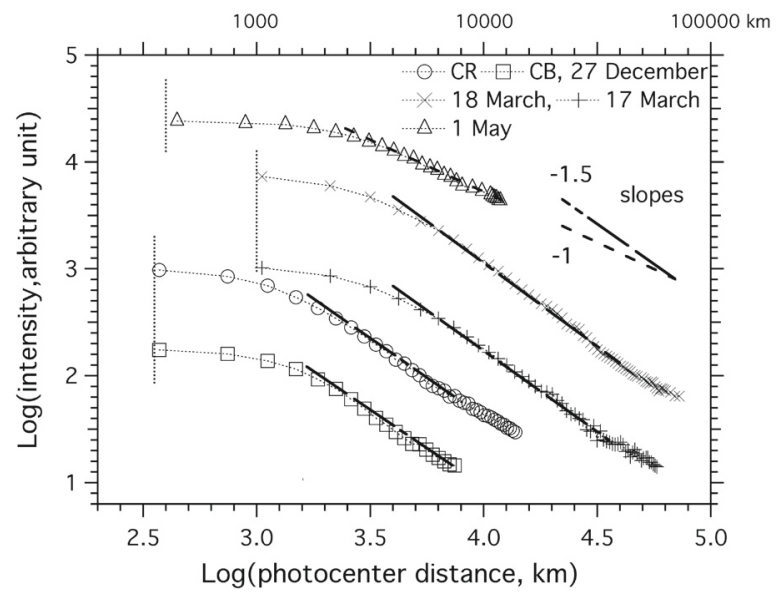

Fig. 1. Radial profiles for the 3 observational periods. Vertical dotted lines indicate the seeing radius limits for each period (less than $330 \mathrm{~km}$ in December, $1050 \mathrm{~km}$ in March, and $450 \mathrm{~km}$ in April-May), but the profiles are not represented in the blurred inner regions.

The same procedure was applied to the three series of observations after separation of the four polarized components. Intensity images were obtained by adding two individual images: either $\left(Z_{0}+Z_{90}\right)$ or $\left(Z_{45}+Z_{135}\right)$. The successive polarized component images with the same orientation and the successive intensity images have to be similar. Because the comet is faint, the signal-to-noise ratio is increased through building each polarized image by adding the individual images for each orientation of the fast axis. This method was systematically used for the OHP observations. It was not always possible to use it for the December observations at IGO, because some technical problems with the rotation of HWP did not allow the use of the whole data set.

Finally, all the polarized components were added to build the intensity image. The radial decrease in intensity was studied first. On the image, the intensity was integrated in increasing apertures. An average intensity value was calculated for each distance to the photocenter by dividing the intensity in one pixel thickness annulus by the number of pixels in the annulus (radial profiles in Fig. 1). 

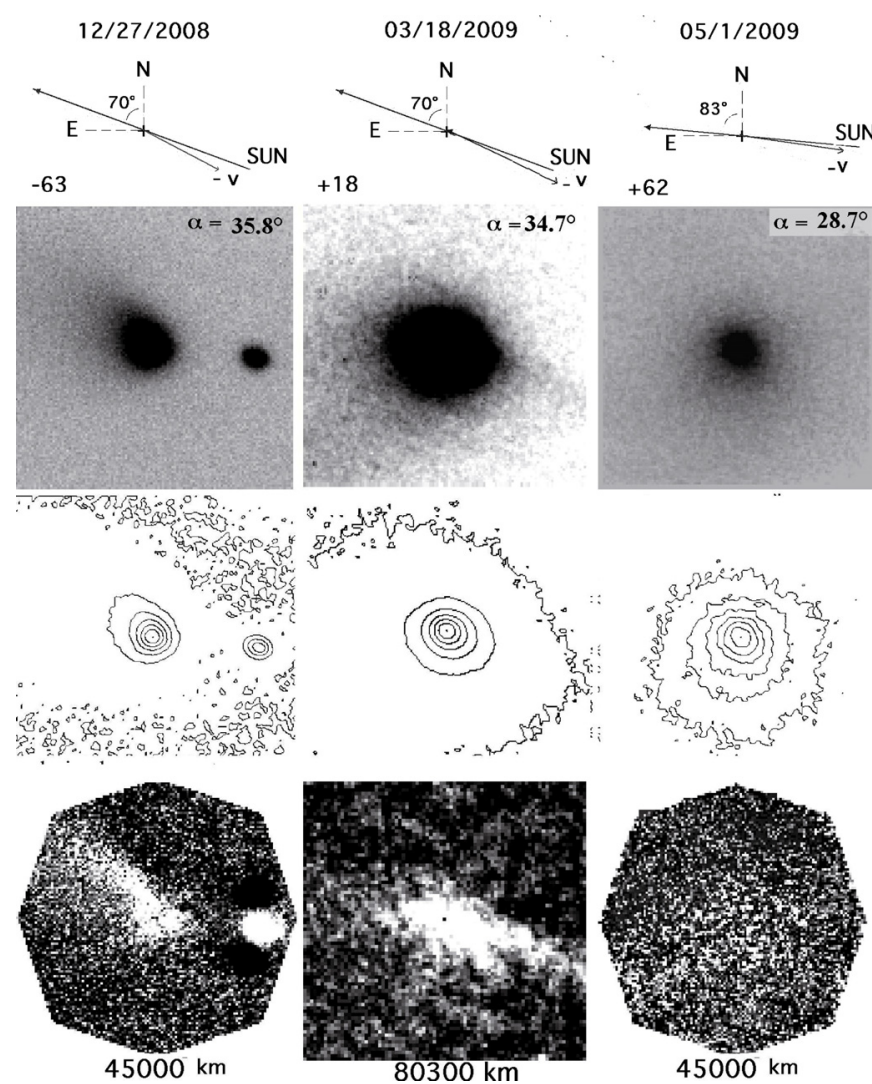

Fig. 2. Dust coma of $67 \mathrm{P} / \mathrm{C}-\mathrm{G}$ in the 2008-2009 apparition pre- and post-perihelion. Horizontal panel 1 (upper): orientation with Sun and star movements from JPL's ephemeris; days to perihelion. Panel 2: intensity images (negative). Panel 3: Isophotes for intensities with the same log scale. Panel 4 (lower): rotational gradient treated images.

Horizontal panel 1 in Fig. 2 presents the geometry of the observations for the three periods, together with Sun and stars movement directions. It indicates that the cometary orbit plane is close to the ecliptic plane in projection on the sky. Intensity images (in negative) are presented in horizontal panel 2, Fig. 2. The main dust features are observed: in December in a cone towards the antisolar direction, in March in a southeast fan prolonged by an antisolar structure and a spike in the solar direction. In April-May, a faint dust ejection is detected all around the photocenter with a slight increase mainly in the southern direction. To better study the intensity decrease with increased photocentric distance, isophotes are drawn with a log scale (panel 3, Fig. 2). To emphasize the high gradient regions on the intensity images (as commonly used to find jet features), treated intensity images were also built (panel 4, Fig. 2) by a rotational gradient method (Larson and Sekanina 1984). Cuts in the intensity images show the decrease in intensity through the different regions of the coma (Fig. 3).

Polarization values through circular apertures centered on the photocenter in the polarized components images were obtained by applying formula (2) and compared to other comets' polarization values (Fig. 4). Finally maps of polarization were obtained by combining four consecutive individual polarized images (Fig. 5). The polarization position angle $\theta$ is calculated by formula (3) or (6). Since the 0 position of one of the polarized filter or of HWP is not systematically aligned with the northsouth celestial direction or with solar direction, the $\theta_{0}$ angle has to be measured on standard stars for each observational period, in order of retrieving $\left(\theta-\theta_{0}\right)$.
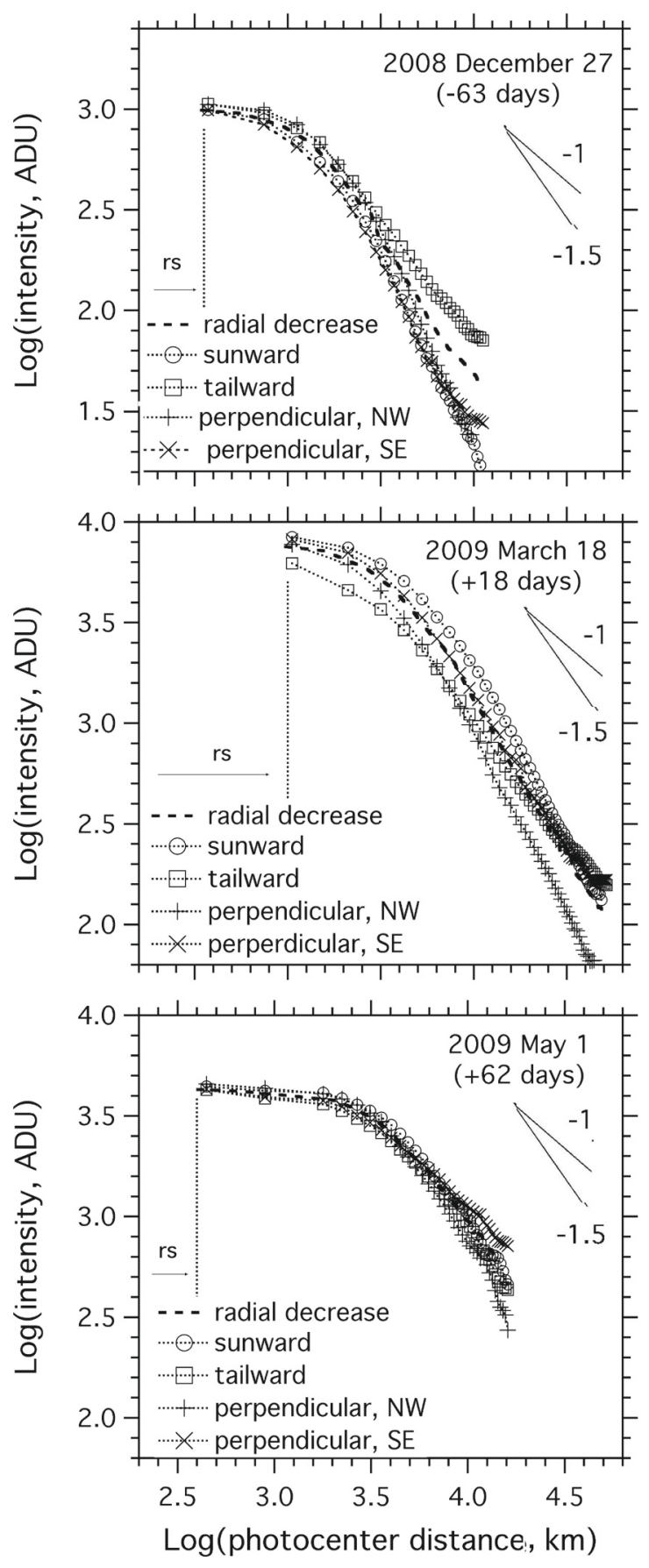

Fig. 3. Cuts through the coma sunward, tailward and in perpendicular directions for the 3 periods of observations. Slopes of -1 and -1.5 are indicated on the graphs. Vertical doted lines indicate the seeing radius (rs) limits for each period, the profiles are not represented in the blurred inner regions

\subsection{Log of the observations}

The log of the observations and the characteristics of filters can be found in Table 1 . The heliocentric distance range is relatively narrow within 1.45 to $1.26 \mathrm{AU}$. The phase angle does not change significantly from pre- to post-perihelion observations, but it only decreases from about $37^{\circ}$ to $29^{\circ}$ post-perihelion. Before perihelion, narrow band and broadband continuous filters were used, while post-perihelion broadband filters were used. In December and April-May the seeing was smaller than 0.8 arcsec, but in March it was smaller than 1.5 arcsec. On May 1, 
Table 2. Standard $\operatorname{stars}^{b}$.

\begin{tabular}{ccccccc}
\hline \hline & Star & $P(\%)$ & PA $\left(^{\circ}\right)$ & $P_{\text {obs }}\left({ }^{\circ}\right)$ & PA $_{\text {obs }}\left({ }^{\circ}\right)$ & $\theta_{0}\left(^{\circ}\right)$ \\
\hline IGO & GD319 & $0.09 \pm 0.09$ & 140 & $0.6 \pm 0.3$ & 113 & $27 \pm 10$ \\
December & C191B2B & $0.09 \pm 0.06$ & 157 & $0.2 \pm 0.1$ & 131 & \\
2008 & HD25443 & $5.13 \pm 0.06$ & 134 & $4.0 \pm 0.5$ & 111 & \\
& HD94851 & $0.06 \pm 0.02$ & NA & $0.2 \pm 0.1$ & 22.5 & \\
& HD251204 & $4.04 \pm 0.07$ & 147 & $2.4 \pm 1.1$ & 117 & \\
OHP & GD319 & $0.09 \pm 0.09$ & 140 & $0.06 \pm 0.05$ & 134 & $-4 \pm 10$ \\
March & HD251204 & $4.04 \pm 0.07$ & 147 & $4.4 \pm 0.2$ & 153 & \\
2009 & HD155197 & $4.38 \pm 0.03$ & 103 & $4.2 \pm 0.15$ & 117 & \\
& HD155197 & $4.38 \pm 0.03$ & 103 & $3.9 \pm 0.4$ & 118 & \\
IGO & & & & & & \\
April- & HD94851 & $0.06 \pm 0.02$ & NA & $0.33 \pm 0.08$ & 67 & $0 \pm 10$ \\
May & HD155197 & $4.46 \pm 0.03$ & 32 & $4.17 \pm 0.2$ & 42 & \\
2009 & HD155197 & $4.38 \pm 0.03$ & 103 & $4.57 \pm 0.07$ & 95 & \\
\hline
\end{tabular}

Notes. ${ }^{(b)}$ Polarization $P$ and position angle PA for the literature values. $P_{\mathrm{obs}}$ and $\mathrm{PA}_{\mathrm{obs}}$ for the measurements. The chosen values for $\mathrm{PA}_{\mathrm{obs}}$ are the closest to the expected ones. NA $=$ not available.

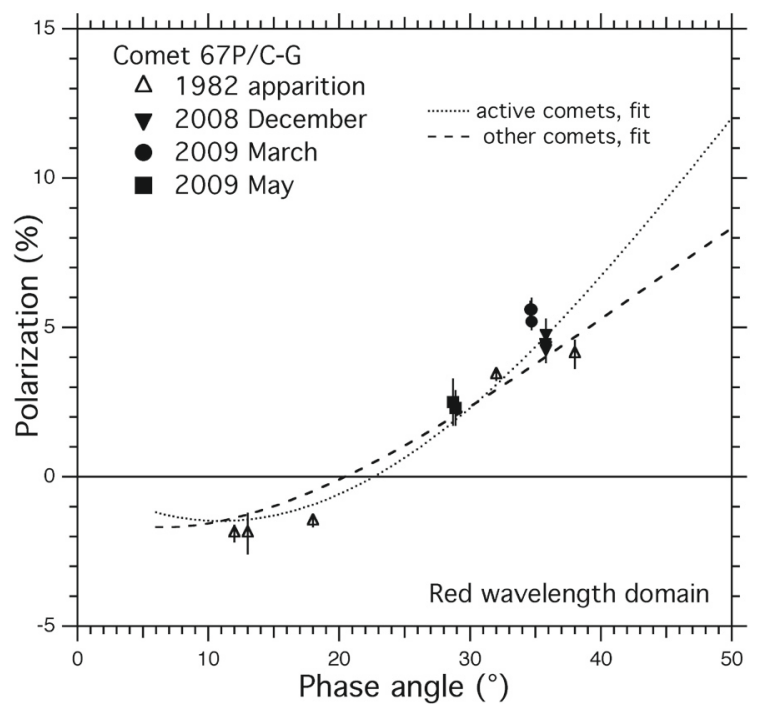

Fig. 4. Comet 67P/C-G polarization observations on 2008-2009 return, as compared to its 1982 apparition (Myers \& Norsiedk 1984) and to synthetic polarization phase curves for other comets.

comet $67 \mathrm{P} / \mathrm{C}-\mathrm{G}$ was only observable for less than one hour, and some star trails were present.

\subsection{Standard stars}

Polarization standard stars were observed during all observational periods to control the non-polarization of the systems and to measure the position angle $\theta_{0}$ of the fast axis of the polarization filters or of HWP (Table 2). The polarization values are similar to values obtained in previous publications (Bastien et al. 1988; Turnshek et al. 1990; Schmidt et al. 1992). Position angles are not completely defined after calculations. Different measurements for the same period help to estimate $\theta_{0}$, and are indicated in the last column of Table 2.

\section{Results}

The intensity variations are first presented in this section, through slopes of intensity radial profiles and comparison with

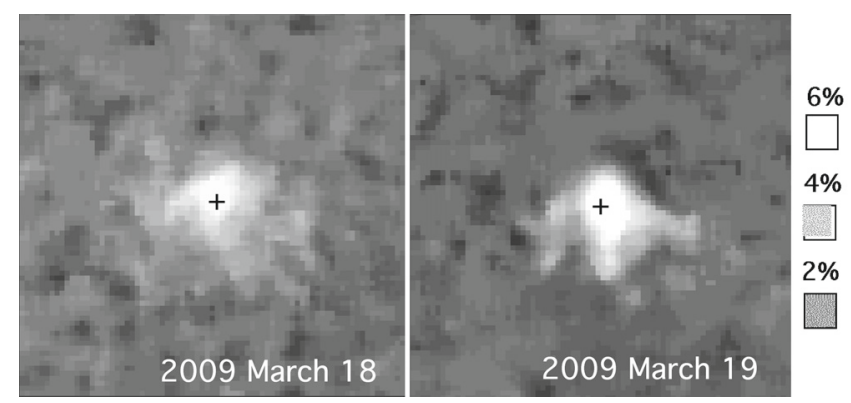

Fig. 5. Polarization map in March 2009. FOV: $40000 \mathrm{~km} .+=$ Photocenter.

an isotropic coma. The different coma morphologies are then presented and important asymmetries pointed out for the first two periods of observations. To have a more precise description of these asymmetries, cuts in the solar-antisolar directions and in the perpendicular directions are made. Finally, variations in the polarization are pointed out.

\subsection{Intensity images}

\subsubsection{Intensity radial profile}

The decrease in intensity as a function of photocentric distance in log-log scale is on average close to -1 for distances up to about $20000 \mathrm{~km}$ for December observations and up to about $60000 \mathrm{~km}$ for March observations Nevertheless, this decrease is not uniform. Close to the photocenter, the slope is dominated by the seeing (less than 2 pixels for each observational period). Farther away, the radial decrease is small (slope between -0.7 and -0.8$)$, but values such as -1.5 are obtained pre- and postperihelion between $2000 \mathrm{~km}$ and $8500 \mathrm{~km}$ in December and between $4500 \mathrm{~km}$ and $40000 \mathrm{~km}$ in March (Fig. 1). The slope of the decrease is -1 at distances greater than $10000 \mathrm{~km}$ in December and more than $50000 \mathrm{~km}$ in March. The strong radial decrease in intensity is noticed through the whole set of filters (narrow blue and red, broad red). In April-May observations, the slope of the radial decrease is -1 (as for an isotropic coma), except in the inner coma where it is very small $(-0.4$ between 2000 and $3500 \mathrm{~km}$, i.e. more than four times the seeing radius). 
In the December and March radial profiles, the steep decrease in intensity is limited in space to an increasing photocentric distance (respectively $8500 \mathrm{~km}$ and $40000 \mathrm{~km}$ ). It may originate in a change of geometry or in an outer displacement of the region where this steep decrease is noticed. In the latter case, the velocity of the particles in projection on the sky is tentatively estimated to $(4.5 \pm 2) \mathrm{ms}^{-1}$ (by dividing the increase in the photocentric distance at the change in slope by the time between the observations).

\subsubsection{Coma morphologies}

In Fig. 2 intensity, isophotes, and treated intensity images (Larson-Sekanina) are presented. The isophotes are in log scale for the intensity, with identical steps for the three periods. The coma is clearly asymmetric in December and March. In December, a large tailward structure $\left(\mathrm{PA}=70^{\circ}\right)$ is noticed, that could be the end edge of a cone (PA between $70^{\circ}$ and $130^{\circ}$ ). For March observations, a bright structure at about $\mathrm{PA}=250^{\circ}$ is observed in the antisolar direction (prolonged by a tailward structure at $\mathrm{PA}=80^{\circ}$ ). At the same period, dust is also ejected in a region between $\mathrm{PA}=90^{\circ}$ and $\mathrm{PA}=230^{\circ}$ (southeast). Several jet features are present in all the images through all filters. For April-May observations, the coma appears quasicircular in projection on the sky with some dust towards the south-southeast direction. On the isophotes, the inner coma appears slightly elongated in the sunward direction. In the treated images, a faint feature seems to be present in the solar direction and in the southeast.

In March, the magnitude (11-12) is smaller by about 2 than its expected value on the ephemeris. Amateur astronomers (e.g. Seiichi 2009) have also observed an increase in intensity, which was still observable by the middle of March. It decreases progressively and is no longer observable at the end of April. An outburst may be suspected to be responsible for this increase.

\subsubsection{Profiles through the intensity images}

In Fig. 3, the variation in the intensity is more precisely studied by cuts through the intensity images. The average radial decrease is also presented for comparison (measured in the same images than the different cuts). The coma, as described in Sect. 3.1 for the two first periods, is highly asymmetric. Nevertheless for April-May observations, the intensity decrease also depends on the direction, and the apparent featureless coma might only result from a geometric effect.

The central regions are affected by the seeing (less than one pixel radius for each period, corresponding to $330 \mathrm{~km}$, $1050 \mathrm{~km}$, and $450 \mathrm{~km}$ ). Farther away, at larger distances but less than about $2200 \mathrm{~km}$ in December and $4200 \mathrm{~km}$ in March, the slopes increase progressively. The low values except in March in the southeast direction (fan direction) may be partially due to the seeing but also to particles moving slowly away from the nucleus. For distances in the $2200 \mathrm{~km}$ to $8500 \mathrm{~km}$ range in December and from $4200 \mathrm{~km}$ to $40000 \mathrm{~km}$ in March, the slopes are steep except in the antisolar direction, where they have an average value of -1.2 in December and March, decreasing to -1 in April-May. In March, in the antisolar direction, the distance interval may be separated in two parts: (4200-16000) km with a slope of -1.3 and (16000-40000) km with a slope of -1.1 . On March 17 and 19, for all directions, the distances and slopes are similar to those obtained on March 18.
The asymmetry of the coma is characterized well by the differences in the slopes for each period. The coma is limited to relatively small distances in the northwest direction. In December, the decrease is very important in the solar direction. In March, it is slightly smaller due to the feature in the antisolar direction, limited to $40000 \mathrm{~km}$ projected distance. In March, the slope in the southeast direction $(-1.4)$ is less steep than in the solar direction, probably because of the emission of fresh dust. An interpretation in terms of properties of the particles is given in Sect. 4.1.

\subsection{Linear polarization}

Aperture polarization corresponds to the integrated flux through apertures measured in the images. The influence of the aperture size is studied, and the values derived from narrow and broad-band filters are compared. Polarization values as a function of phase angle are characteristic of cometary-dust's average physical properties. The data are thus compared to previous phase curves obtained for comet $67 \mathrm{P} / \mathrm{C}-\mathrm{G}$ and for other comets (see Sect. 4). In the polarization maps, regions are tentatively detected where dust is freshly ejected from the nucleus and has different physical properties than those of the surrounding particles.

\subsubsection{Aperture polarization}

The polarization through increasing aperture sizes is presented in Table 3 for the different filters and periods. The polarization phase curve is presented in Fig. 4.

Only small changes in polarization values with aperture are observed through the filters (narrow or broadband). For December observations, there is no difference in polarization between the red and blue wavelengths (cometary narrow filters and red broad filter) at these intermediate phase angles $\left(35^{\circ}\right)$ for this dust rich comet. These results confirm the negligible contaminations by the emission lines of the gaseous species through the broadband filters. The relatively large variations in polarization in the inner coma $(D \leq 3000 \mathrm{~km})$, up to $1 \%$ in March can hardly be attributed to the nucleus rotation, since they are within the error bars (average value $5.8 \pm 0.4$ ).

The polarization after perihelion is clearly higher than before perihelion, while the phase angle has decreased. Such an increase in polarization is likely to be the result of dust ejected during the outburst some days after perihelion correlated to the increase in intensity (Sect. 3.1.2). Two months after perihelion (April-May), the polarization has decreased as expected with the decrease in phase angle, although it may be slightly above the remaining value.

\subsubsection{Polarization maps}

Polarization maps are retrieved for the two first periods. For the April-May observations, as noticed in Sect. 2.3, star trails are too close to the photocenter to retrieve a polarization map. Sixty days before perihelion (December), the polarization map is quite uniform in a field of view of $12000 \mathrm{~km}$ diameter, without any feature, indicating that there is no major difference in the physical properties of the dust between the tailward structure and the other parts of the coma, including the region surrounding the photocenter.

Three weeks after perihelion the polarization close to the photocenter is about $6 \%$. The polarization in the features is (4-6)\% for a surrounding polarization of $(2-3) \%$. The features are mainly found in the south and sunward directions but also 
Table 3. Polarization in percent as a function of aperture diameter ${ }^{c}$.

\begin{tabular}{cccccc}
\hline \hline $\begin{array}{c}\text { Date } \\
\text { 2008-2009 }\end{array}$ & $\begin{array}{c}D \\
\text { Filters }\end{array}$ & $\begin{array}{c}3000 \\
(\mathrm{~km})\end{array}$ & $\begin{array}{c}6000 \\
(\mathrm{~km})\end{array}$ & $\begin{array}{c}12000 \\
(\mathrm{~km})\end{array}$ & $\begin{array}{c}24000 \\
(\mathrm{~km})\end{array}$ \\
\hline $\begin{array}{c}\text { December } \\
25.60\end{array}$ & $\mathrm{CR}$ & $4.0 \pm 0.7$ & $4.5 \pm 0.4$ & $4.5 \pm 0.5$ & $S / N$ \\
26.62 & $\mathrm{CR}$ & $4.1 \pm 0.8$ & $4.8 \pm 0.5$ & $4.8 \pm 0.8$ & $S / N$ \\
27.59 & $\mathrm{CR}$ & $4.2 \pm 0.3$ & $4.4 \pm 0.3$ & $4.4 \pm 0.3$ & $S / N$ \\
27.59 & $\mathrm{CB}$ & $4.4 \pm 0.5$ & $4.5 \pm 0.5$ & $4.5 \pm 0.5$ & $S / N$ \\
27.63 & $R_{\mathrm{b}}$ & $4.5 \pm 0.3$ & $4.4 \pm 0.5$ & $3.9 \pm 0.5$ & $3.8 \pm 0.8$ \\
March & & & & & \\
17.81 & $R$ & $5.2 \pm 1.0$ & $5.1 \pm 0.3$ & $5.6 \pm 0.4$ & $5.8 \pm 0.5$ \\
18.78 & $R$ & $6.3 \pm 0.9$ & $4.9 \pm 0.3$ & $5.2 \pm 0.3$ & $5.2 \pm 0.3$ \\
19.80 & $R$ & $5.9 \pm 1.1$ & $5.8 \pm 0.5$ & $5.6 \pm 0.3$ & $5.4 \pm 0.3$ \\
April-May & & & & & \\
30.60 & $R_{\mathrm{b}}$ & $2.7 \pm 0.8$ & $2.5 \pm 0.8$ & $2.5 \pm 1.1$ & $S / N$ \\
01.70 & $R_{\mathrm{b}}$ & $2.5 \pm 0.5$ & $2.3 \pm 0.5$ & $2.3 \pm 0.8$ & Star trails \\
\hline
\end{tabular}

Notes. ${ }^{(c)} D=$ projected diameter. Filters: see Table 1 caption. $S / N$ : signal over noise ratio $\leq 3$. Star trails in the cometary coma. In the error bars, systematic errors (subtraction of the background, error bars on the standard stars) are included.

tailward. Figure 5 presents the polarization map for 18 and 19 March observations. A median filter was applied to the images. The higher polarization regions are correlated to the features observed on the rotational gradient intensity images (Fig. 2). The main features have the same orientation on the two dates, but they seem to be more extended on March 19.

The angle $\theta_{0}$ between the polarization vector and the fast axis of filter 0 at each observational period is quasi-uniform on the whole field, without any measurable deviation and equal to $(122 \pm 15)^{\circ}$ in December, $(159 \pm 10)^{\circ}$ in March, and $(77 \pm 15)^{\circ}$ in May. The angles $\theta-\theta_{0}$ between the polarization vector and the solar direction are equal to $(95 \pm 15)^{\circ},(91 \pm 10)^{\circ}$ and $(77 \pm$ $20)^{\circ}$. As expected, the results lead to a polarization vector perpendicular to the solar direction.

The polarization maps are different in March as compared to December, indicating dust with physical properties closer to those usually observed for active comets.

\section{Discussion}

\subsection{Comparison between the different periods of observation}

The different changes in morphology may stem from changes in the orientation of the rotational axis of the nucleus as compared to the Sun position (seasonal effects). Before perihelion (about 2 months), the sunward feature may correspond to a rotating jet ejecting material in a cone with its side-end on the northeast direction. It might also come from dust slowly pushed back in the tail direction. The absence of change in polarization indicates that, on average, the physical properties of the particles remain similar inside the whole coma. The significant decrease in the slope of the intensity around the photocenter may be due to large particles mainly concentrated at short distances to the nucleus and not too sensitive to the solar radiation pressure.

After perihelion (about 3 weeks), the sunward structure may correspond to an antitail (perspective view, see e.g. Agarwal et al. 2007b). Nevertheless, the polarization variations inside the coma indicate that particles ejected in the southeast direction in a large fan present different physical properties, the antitail consisting mainly of large, previously ejected particles. A part of the dust seems to be sensitive to the solar radiation pressure; it can be made of smaller grains (submicron or micron-sized) eventually in fluffy aggregates with a higher velocity than the large more compact particles (larger than hundreds of micrometers). From March 18 to March 19, the structures seem to extend mainly to the south (Fig. 5).

The absence of major features in April-May (except perhaps in the solar and southeast directions) may correspond to a change in geometry, as well as to a decrease in activity at increasing heliocentric distances. The slopes, close to $(-1)$, suggest that the very large grains are no longer predominant in the coma. The absence of jet features suggests a quiet coma mainly made up of small grains probably in fluffy aggregates.

\subsection{Comparison with previous observations of $67 P / C-G$}

\subsubsection{Intensity}

Such steep slopes as those observed before perihelion and just after was also previously observed during the 82-83 and 95-96 apparitions (Schleicher 2006). A post-perihelion outburst was noticed about one month after perihelion for the 1996-1997 and 2002-2003 apparitions (Kidger 2003). On the secular light curve, a decrease in activity (intensity decrease) usually appears about 100 days before perihelion (Schulz et al. 2004; Ferrin 2005). Post-perihelion, the envelope of the light curve remains the same as the inbound leg whenever the comet activity is continuously detected. The maximum in intensity actually occurs about one month after perihelion. The magnitude variations that we observe seem to be the result of a seasonal effect and not of an outburst with a sudden decrease in magnitude.

Tozzi et al. (2009) have observed 67P/C-G before perihelion at different decreasing heliocentric distances (3 AU to 1.3 AU). From the variations and evolution of intensity images of the coma, they conclude that very large particles ( $\mathrm{mm}$ to $\mathrm{cm}$-sized) are present, moving very slowly in the coma. They do not find any variation with wavelength related to the presence of the large particles and exclude the presence of sublimating organic grains. The authors estimate the average velocity of the particles (from the progressive extension of the coma) to be in the $0.3 \mathrm{~m} \mathrm{~s}^{-1}$ to $3.5 \mathrm{~m} \mathrm{~s}^{-1}$ range for decreasing heliocentric distance. The velocity we obtain in Sect. 3.1.1 is quite comparable.

Using data available from recent and previous apparitions, Fulle et al. (2010) have modeled the dust environment of the comet and estimate the evolution of tail, trail, and antitail. Their conclusions are similar to those of Tozzi et al. (2009) with emission of $\mathrm{mm}$ to $\mathrm{cm}$-sized particles before perihelion. They 
also suggest a strong seasonal effect with the north pole illuminated by the Sun before perihelion and the south pole after perihelion. From the increase in the velocity of the particles, together with coma and tail models, they conclude it is the emission of small grains post-perihelion from the southern hemisphere. They also suggest that the autumn equinox took place in January 2009, i.e. before perihelion. As suggested in the present work, the particles mainly seem to be large before perihelion, while numerous small grains (probably included in large fluffy aggregates) are ejected at the time of maximum activity, about three weeks after perihelion. Later on, two months after perihelion, the activity decreases, while small grains seem to dominate in the coma (spring on the southern hemisphere).

\subsubsection{Polarization}

The aperture polarization values we obtained for the 2008-2009 apparition are compared to those obtained for other comets and to previous observations of $67 \mathrm{P} / \mathrm{C}-\mathrm{G}$ (Fig. 4). Two main classes of comets have been defined by their aperture polarization. At larger phase angles than $(30-40)^{\circ}$, active comets with well-confined jets present a higher polarization than less active comets (see e.g. Hadamcik \& Levasseur-Regourd 2003a). The polarization values measured before perihelion on 2008 , December are between the two synthetic curves corresponding to trigonometric fits on all the available data for active comets and for other comets (they are very close to one another at phase angles smaller than $40^{\circ}$ ). The values obtained about three weeks after perihelion in March are above the curves by about $1 \%$. The April-May values are just above the curves by about $0.5 \%$, but the difference is within the error bars so it is difficult to decide. As noticed in Levasseur-Regourd et al. (2004), the polarization values obtained by Myers \& Nordsieck (1984) and Chernova et al. (1993) are close to the synthetic phase curves but not on them (Fig. 4). Myers and Nordsieck used a spectropolarimeter, possibly leading to small differences with results from aperture polarimetry (some coma regions are not in the slit). The data corresponding to their observations six weeks before perihelion and about 2.5 months after are below the synthetic phase curves by approximately $1 \%$. The value obtained on 1982, November 17, ten days after perihelion, is just above the curves by about $0.5 \%$ and corresponds to an increase of about $1.5 \%$ over Myers \& Nordsieck (1984) phase curve. Chernova et al. (1993) observed the comet three weeks after perihelion by aperture polarimetry in the blue domain and at a phase angle of $27.2^{\circ}$, and their value corresponds to a polarization level similar to our April-May observation in the red domain at $28.8^{\circ}$. Indeed, no noticeable color effect has been noticed neither in our observations nor in Myers $\&$ Nordsieck (1984) observations. For all polarization measurements, except perhaps post-perihelion, 67P/C-G may be classified as a low $P_{\max }$ comet, which is typical of JFCs (Kolokolova et al. 2004) with large dark particles. This classification agrees with the absence of silicate features in the infrared at wavelengths close to $10 \mu \mathrm{m}$ for observations made pre-perihelion in 1982 (Hanner et al. 1985).

The dust ejected after perihelion seems to be more polarized than before perihelion. Moreover, some features present on the polarization maps are reminiscent of those observed in more active comets. The dust is pushed towards the tail with a faster speed than before perihelion. In projection on the sky, the smaller grains are seen in the same line of sight as the large grains ejected before perihelion. Such smaller grains may be the constituents of large fluffy aggregates.

\subsection{Comparison to other Jupiter family and active comets}

Albedo values of $67 \mathrm{P} / \mathrm{C}-\mathrm{G}$ dust obtained during previous apparitions are summarized in Hadamcik and Levasseur-Regourd (2009) along with their phase angle variation. They are compared to those obtained for some JFCs and the comet 1P/Halley. Geometric albedo values are found in the 0.04 and 0.06 range (Fig. 5 in Hadamcik \& Levasseur-Regourd 2009, and references therein). Fulle et al. (2010) suggest an albedo between 0.05 and 0.06 . This very low albedo and the presence of large fluffy aggregates can be the reason for the constant (or slightly increased) polarization through large apertures mainly as three weeks post-perihelion. Indeed, large aggregates induce a slight increase in polarization whenever fragmentation of the aggregates take place (see e.g. Hadamcik et al. 2009, for the experimental simulations).

In spite of the relative faintness of $67 \mathrm{P} / \mathrm{C}-\mathrm{G}$ during its last return, activity is obvious from coma asymmetries and features. Within the $29^{\circ}-36^{\circ}$ phase angle range, significant differences in polarization cannot be expected. However, the post-perihelion increase in polarization is a clue to the presence of smaller grains in fast-moving particles for the freshly emitted dust. Such an increase has also been observed for other comets (see e.g. Dollfus et al. 1988, for 1P/Halley; Tozzi et al. 1997, for C/1996 B2 Hyakutake; Hadamcik \& Levasseur-Regourd 2003c, for C/1999 S4 LINEAR). In the two last cases, they were observed when the nucleus broke down into smaller fragments ejecting numerous fragmenting particles and gas, which does not seem to be the case for comet $67 \mathrm{P} / \mathrm{C}-\mathrm{G}$.

A comparison with comet $26 \mathrm{P} /$ Grigg-Skjellerup is of interest, since remote observations by Jockers et al. (1994) of this relatively faint comet were modeled by Fulle et al. (1994) and since this comet was observed in-situ by the Optical Probe Experiment "OPE" onboard the Giotto spacecraft (LevasseurRegourd et al. 1993). In the inner coma (closer distance to nucleus about $270 \mathrm{~km}$ ), strong intensity and polarization variations were observed (Renard et al. 1996). The color and polarization variations allowed these features to be interpreted as jets and as a possible small fragment. From the intensity gradients, McBride et al. (1997) modeled the dust profile and confirmed the results relative to the presence of jets and of a small fragment of $10-100 \mathrm{~m}$ at about $1000 \mathrm{~km}$ from the nucleus. The OPE brightness results have been degraded to the ground-based resolution and geometry, leading to a perfect agreement with Fulle's model results.

Comet $81 \mathrm{P} /$ Wild 2 is a dust-rich, relatively active comet for which, like 67P/C-G, only some of the features have counterparts on the polarization maps (Hadamcik \& Levasseur-Regourd 2003a). Indications for the dust properties have been obtained by the in situ Dust Flux Monitor onboard Stardust (Tuzzolini et al. 2004). Strong local variations were observed, some of them interpreted by swarms of fragmenting fluffy aggregates (Clark et al. 2004). Particles captured on aerogels in the coma are indeed either large compact grains or fluffy aggregates of tiny grains (Hörz et al. 2006). The later type may be present postperihelion in $67 \mathrm{P} / \mathrm{C}-\mathrm{G}$ coma, with a relative abundance increasing with time (seasonal effect).

As in the case of $67 \mathrm{P} / \mathrm{C}-\mathrm{G}$, the polarization value retrieved for comet $9 \mathrm{P} /$ Tempel 1 before the Deep Impact event was between the values corresponding to the two synthetic curves (Hadamcik et al. 2007a). Variations in polarization were observed in the inner coma of $67 \mathrm{P} / \mathrm{C}-\mathrm{G}$ (Sect. 3.2.1). These variations cannot be related with certainty to the nucleus rotation as for $9 \mathrm{P}$, for which a strong decrease in intensity was also observed 
in some directions as well as an important polarization decrease with increasing distance to the photocenter. During the Deep Impact event, small fast-moving grains were found to be ejected into the coma. The increase in intensity during the ejection was sudden. It was progressive for comet $67 \mathrm{P} / \mathrm{C}-\mathrm{G}$, but a similar increase in polarization was observed. The fast-moving grains ejected by Deep Impact were likely to originate in the subsurface. A similar origin may be suggested for the grains coming from the southern hemisphere of comet $67 \mathrm{P} / \mathrm{C}-\mathrm{G}$.

\section{Conclusions}

Complementary light-scattering observations of the coma of comet 67P/C-G were performed in France and India during three periods: on 2008 December, two months before perihelion, on 2009 March, three weeks after perihelion and on 2009 April-May, two months after perihelion. They allowed to conclusions on a change in the physical properties of the ejected dust related to seasonal variations. From the steep radial decrease in intensity before perihelion and just after, large, slowly moving compact dark particles seem to be present in the coma. The absence of any feature on the polarization map indicates that the dust properties are on average the same in all directions. After perihelion, a correlated increase in intensity and polarization (as compared to before perihelion) suggests the emission of fluffy aggregates of small grains. All these results confirm the results obtained by different techniques during previous apparitions indicating that the main physical properties of the dust do not change from one apparition to the next. The coma seems to be dominated by large grains originating in one hemisphere pre-perihelion and by small grains from the other hemisphere post-perihelion.

Comet $67 \mathrm{P} / \mathrm{C}-\mathrm{G}$ is a dust-rich Jupiter-family comet. By its restricted activity, it can be compared to comet $26 \mathrm{P} / \mathrm{Grigg}$ Skjellerup. It is similar to comet $9 \mathrm{P} /$ Tempel 1 because of the large particles emitted before perihelion. It is also similar to comet $81 \mathrm{P} /$ Wild 2 because of the likely presence of fluffy aggregates after perihelion. The surface or subsurface origin of the particles will only be given by the Rosetta in situ measurements. All along its rendezvous with 67P/C-G, Rosetta should encounter dust particles consisting of large conglomerates that are only observable from the spacecraft (MIDAS, GIADA detection).

Acknowledgements. We thank Daniel Bardin for his help in the observations at OHP. We gratefully acknowledge ESA for the narrow-band filters at IGO. OHP and IUCAA are acknowledged for allocation of observation time, PNP for funding the French observations. This is Jeremie Lasue contribution number 1542 for the LPI.

\section{References}

A’Hearn, M. F., Millis, R. L., Schleicher, D. G., Osip, D. J., \& Birch, P. V. 1995, Icarus, 118, 223

Agarwal, J., Boenhardt, H., \& Grün, E. 2007a, ESA-SP, 643, 51

Agarwal, J., Müller, M., \& Grün, E. 2007b, Space Sci. Rev., 128, 79

Bastien, P., Drissen, L., Menard, F., et al. 1988, AJ, 95, 900

Chernova, E. P., Kiselev, N. N., \& Jockers, K. 1993, Icarus, 103, 144
Clark, B. C., Green, S. F., Economou, T. E., et al. 2004, J. Geophys. Res., 109, E12S03

Davidsson, B. J. R., \& Guttiérrez, P. J. 2005, Icarus, 176, 453

Dollfus, A., Bastien, P., Le Borgne, J. F., Levasseur-Regourd, A. C., \& Mukai, T. 1988, A\&A, 206, 348

Ferrin, I. 2005, Icarus, 178, 493

Fulle, M., Barbieri, C., Cremonese, G., et al. 2004, A\&A, 422, 357

Fulle, M., Colangeli, L., Agarwal, J., et al. 2010, A\&A, in press

Glassmeier, K. H., Boehnardt, H., Koshny, D., Kuhrt, E., \& Richter, I. 2007, Space Sci. Rev. 128, 1

Hadamcik, E., \& Levasseur-Regourd, A. C. 2003a, J. Quant. Spec. Radiat. Transf., J. Quant. Spec. Radiat. Transf., 79, 661

Hadamcik, E., \& Levassseur-Regourd, A. C. 2003b, A\&A, 403, 757

Hadamcik, E., \& Levasseur-Regourd, A. C. 2003c, Icarus, 166, 188

Hadamcik, E., \& Levasseur-Regourd, A. C. 2009, Planet. Space Sci., 57, 1118

Hadamcik, E., Renard, J.-B., Levasseur-Regourd, A. C., \& Lasue, J. 2006, J. Quant. Spec. Radiat. Transf., 100, 143

Hadamcik, E., Levasseur-Regourd, A. C., Leroi, V., \& Bardin, D. 2007a, Icarus, 190,459

Hadamcik, E., Renard, J.-B., Rietmeijer, F. J. M., et al. 2007b, Icarus, 190, 660 Hadamcik, E., Renard, J.-B., Levasseur-Regourd, A. C., et al. 2009,

J. Quant. Spec. Radiat. Transf., 110, 1755

Hanner, M. S., Tedesco, E., Tokunaga, A. T., et al. 1985, Icarus, 64, 11

Hörtz, F., Bastien, R., \& Borg, J. 2006, Science, 314, 1716

Kelley, M. S., Reach, W. T., \& Lien, D. L. 2008, Icarus, 193, 572

Kidger, M. R. 2003, A\&A, 408, 767

Kolokolova, L., Hanner, M. S., Levasseur-Regourd, A. C., \& Gustafson, B. 2004, in Comet II, ed. M. Festou, H. U. Keller, \& H. A. Weaver, 577

Lamy, P. L., Toth, I., Weaver, H., Jorda, L., \& Kassalainen, M. 2003, BAAS, 35, 970

Lamy, P. L., Toth, I., Groussin, O., et al. 2008, A\&A, 489, 777

Larson, S., \& Sekanina, Z. 1984, AJ, 89, 571

Lasue, J., Levasseur-Regourd, A. C., Hadamcik, E., \& Alcouffe, G. 2009, Icarus, 199, 129

Levasseur-Regourd, A. C. 1999, Space Sci. Rev., 90, 163

Levasseur-Regourd, A. C., \& Hadamcik, E. 2003, J. Quant. Spec. Radiat. Transf., 79, 903

Levasseur-Regourd, A. C., Bertaux, J. L., Dumont, R., et al. 1986, Nature, 321, 341

Levasseur-Regourd, A. C., Goidet, B., Le Duin, T., et al. 1993, Planet. Space Sci., 41, 167

Levasseur-Regourd, A. C., McBride, N., Hadamcik, E., \& Fulle, E. 1999, A\&A, 348,636

Levasseur-Regourd, A. C., Hadamcik, E., Lasue, J., Renard, J.-B., \& Worms, J. C. 2004, in The new Rosetta targets, ed. L. Colangeli, E. Mazzotta, E. Epifani, \& P. Palumbo (Dordrecht: Kluwer), 111

Levasseur-Regourd, A. C., Hadamcik, E., Sen, A. K., Gupta, R., \& Lasue, J. 2010, in Icy Bodies of the Solar System, ed. D. Lazzaro, R. Prialnik, R. Schulz, \& J. A. Fernandez, Proc. IAU, 263, accepted

McBride, N., Green, S. F., Levasseur-Regourd, A. C., Goidet-Devel, B., \& Renard, J.-B. 1997, MNRAS, 289, 535

Myers, R. V., \& Nordsiek, K. H. 1984, Icarus, 58, 431

Ramaprakash, A. N., Gupta, R., Sen, A. K., \& Tandon, S. N. 1998, A\&AS, 128, 369

Renard, J.-B., Levasseur-Regourd, A. C., \& Dollfus, A. 1992, Ann. Geophys., 10,288

Renard, J.-B., Hadamcik, E., \& Levasseur-Regourd, A. C. 1996, A\&A, 316, 263 Rocher, P., http://imce.fr/langues/en/ephemerides/donnes/comets. FICH/CIFO029.php

Schleicher, D. G. 2006, Icarus, 181, 442

Schulz, R. 2004, A\&A, 422, L19

Seiichi, Y. 2009, http: //www . aerith.net/index.html

Sen, A. K., Joshi, U. C., Deshpande, M. R., \& Prasad, C. D. 1990, Icarus, 86, 248

Schmidt, G. D., Elston, R., \& Lupie, O. L. 1992, AJ, 104, 1563

Tozzi, G. P., Cimatti, A., di Serego Alighieri, S., \& Cellino, A. 1997, Planet. Space Sci.., 45, 535

Tozzi, G. P., Boehnardt, H., Licandro, J., et al. 2009, BAAS, DPS, 41, 3703

Turnshek, D. A., Boblin, R. C., Willamson, R. L. II, et al. 1990, AJ, 99, 1243

Tuzzolino, A. J., Economou, T. E., Clark, R. C., et al. 2004, Science, 304, 1776 